\title{
Disparition du Pr Marc Lemann
}

\section{Death of Professor Marc Lemann}

\section{R. Laugier - B. Richard-Molard · Conseil d'administration de la SFED}

C) Springer-Verlag France 2010

C'est avec une très grande tristesse que nous avons appris la disparition brutale du Pr Marc Lemann, survenue le 25 août dernier. Expert reconnu des MICI, bien sûr, en France mais aussi internationalement, il a signé de très nombreuses publications scientifiques et a participé à d'innombrables congrès nationaux et internationaux. Marc n'hésitait pas à donner de son temps sans compter pour participer à de multiples formations durant lesquelles nombre de gastroentérologues issus de tous horizons avaient pu apprécier ses dons de pédagogue, son humour et sa grande simplicité qui sous-entendaient l'étendue de ses qualités humaines. Qu'il soit remercié pour tout ce qu'il a apporté à la connaissance des maladies inflammatoires, à toute la gastroentérologie française et à nous tous. Son absence sera lourdement et durablement ressentie, en particulier dans notre monde de la gastroentérologie, et son exemple restera dans nos mémoires. Nous tenons à exprimer dans ces quelques lignes toute notre émotion et à dédier toutes nos pensées les plus chaleureuses à sa famille ainsi qu'à ses proches.

La SFED tient à rendre un hommage tout particulier à Marc Lemann, et une session lui sera consacrée pendant la prochaine édition de Vidéo-Digest et du séminaire de la SNFGE, le vendredi 5 novembre 2010. 